\title{
Protocol
}

\section{Internet-based cognitive behavioral therapy versus internet-based modified present-centered therapy for world trade center responders and survivors with posttraumatic stress disorder: rationale and design of a randomized controlled trial}

\author{
Hannah R. Brinkman ${ }^{1,2 *}$, Mary L. Kowalchyk ${ }^{1}$, Leah Cahn ${ }^{1}$, Cindy J. Aaronson ${ }^{1}$, \\ Maria Böttche ${ }^{3,4}$, Candice Presseau ${ }^{5,6}$, John C. Markowitz ${ }^{7}$, Brett T. Litz ${ }^{5,8,9}$, \\ Laura Huckins $^{1,6,10-13}$, Rachel Yehuda ${ }^{1,6,14}$, Christine Knaevelsrud ${ }^{3}$, \\ Robert H. Pietrzak ${ }^{15,16}$, Adriana Feder ${ }^{1}$
}

\footnotetext{
${ }^{1}$ Department of Psychiatry, ${ }^{10}$ Department of Genetics and Genomic Sciences, ${ }^{11}$ Pamela Sklar Division of Psychiatric Genomics, ${ }^{12}$ Icahn Institute for Genomics and Multiscale Biology, ${ }^{13}$ Seaver Autism Center for Research and Treatment, ${ }^{14}$ Department of Neuroscience, Icahn School of Medicine at Mount Sinai, New York, NY, USA

${ }^{2}$ Department of Psychology, Rutgers, The State University of New Jersey, New Brunswick, NJ, USA

${ }^{3}$ Department of Clinical Psychological Intervention, Freie Universität Berlin, Germany

${ }^{4}$ Center Ueberleben, Berlin, Germany

${ }^{5}$ VA Boston Healthcare System, Boston, MA, USA

${ }^{6}$ Mental Illness Research, Education and Clinical Center, James J. Peters Veterans' Administration Medical Center, Bronx, New York, USA

${ }^{7}$ Department of Psychiatry, Columbia University; New York State Psychiatric Institute, New York, NY, USA

${ }^{8}$ Department of Psychiatry, Boston University School of Medicine, Boston, MA, USA

${ }^{9}$ Department of Psychological and Brain Sciences, Boston University, Boston, MA, USA

${ }^{15}$ Department of Psychiatry, Yale University School of Medicine, New Haven, CT, USA

${ }^{16}$ United States Department of Veterans Affairs National Center for Post-traumatic Stress Disorder, Clinical Neurosciences Division, VA Connecticut Healthcare System, West Haven, CT, USAA
}

Received: 11 March 2021

Revised: 19 April 2021

Accepted: 21 April 2021

*Correspondence:

Hannah R. Brinkman,

E-mail: hannah.brinkman@ rutgers.edu

Copyright: (c) the author(s), publisher and licensee Medip Academy. This is an open-access article distributed under the terms of the Creative Commons Attribution Non-Commercial License, which permits unrestricted non-commercial use, distribution, and reproduction in any medium, provided the original work is properly cited.

\section{ABSTRACT}

Background: Nearly two decades following the 9/11/2001 world trade center (WTC) attacks, a substantial proportion of WTC rescue and recovery workers ("responders") and WTC survivors continue to experience WTC-related posttraumatic stress disorder (PTSD) symptoms. Internet-based cognitive behavioral therapies (I-CBT) are short-term, evidence-based, scalable treatments with the potential to reach large numbers of symptomatic WTC workers and survivors. However, no ICBT studies have been conducted in the WTC cohort.

Methods: This report describes the rationale and design of an ongoing randomized controlled trial comparing integrative testimonial therapy (ITT), an I-CBT, to an active comparison treatment, internet-based modified present-centered therapy The primary aim is to evaluate the efficacy of ITT in mitigating WTC-related PTSD symptoms in WTC responders and survivors with full or subthreshold WTC-related PTSD. The efficacy of ITT in reducing comorbid depressive and anxiety symptoms, and improving functioning, quality of life, and post-traumatic growth will additionally be evaluated. Saliva samples are also collected to explore genetic and epigenetic biomarkers of treatment response.

Conclusions: This is the first I-CBT trial to compare ITT to a credible and active treatment, controlling for critical thirdvariable explanations of superiority (e.g., non-specific therapy effects). This RCT bridges an important research gap in the rising field of I-CBT interventions and adds to the literature on the design of trials investigating evidence-based treatments for PTSD in WTC- and other trauma-affected populations.

Trial registration: This trial was registered on clinicalTrials.gov on May 16, 2017 (NCT03154151).

Keywords: Posttraumatic stress disorder, Internet intervention, Online therapy, 9/11, World trade center 


\section{INTRODUCTION}

Nearly two decades following the 9/11/2001 world trade center (WTC) attacks, a substantial proportion of WTC rescue and recovery workers ("responders") and WTC survivors continue to experience clinically significant symptoms of posttraumatic stress disorder (PTSD). ${ }^{1,2}$ In a recent survey, the prevalence of probable full and subthreshold PTSD in police responders was 9.3\% and $17.5 \%$, respectively, and in non-traditional responders (e.g., construction workers) $21.9 \%$ and $24.1 \%$, respectively. ${ }^{3} \quad$ Studies have also documented the persistent and pervasive impact of the 9/11 attacks on individuals who lived, worked, or were passersby in the areas surrounding the WTC, with rates of screening positive for PTSD ranging from 11.9-15.7\% in the second decade post-9/11. ${ }^{2}$ PTSD symptom chronicity is concerning, as symptomatic WTC responders and survivors have elevated rates of comorbid psychiatric and medical conditions, and impaired social and occupational functioning and quality of life. ${ }^{3,4}$ Yet multi-wave surveys of WTC cohort members suggest mental healthcare utilization among WTC responders and survivors could be improved with $31.1 \%$ of respondents reporting previous unmet mental health care needs, and $43.1 \%$ of respondents screening positive for PTSD not seeking counseling. ${ }^{5}$ Despite initiatives to expand access to mental health services to WTC cohort members via the WTC Health Program (WTC-HP), a government-initiated program established in response to the 9/11 attacks that provides free health monitoring and treatment to eligible WTC responders and survivors, considerable barriers to treatment remain.

While face-to-face specialty care cognitive behavioral therapy (CBT) ranks among the most effective and empirically supported PTSD treatments, its provision to those still suffering from WTC-related PTSD has been limited by geographical distance, insufficient availability of expert therapists, and stigma associated with seeking mental health treatment. Now compounded by the COVID-19 pandemic, access to in-person therapy has essentially been put on hold, ${ }^{6}$ requiring ongoing efforts to increase access to care. Moreover, PTSD is associated with high treatment avoidance, generalized mistrust of others, and one of the longest delays to accepting treatment. ${ }^{7}$ One approach to overcoming these barriers is Internet-based therapy, which is geographically independent and can reduce feelings of stigma through visual anonymity. Research has shown that when compared to waitlist controls, I-CBT significantly reduces PTSD and related symptoms, improves quality of life, and increases posttraumatic growth. ${ }^{8-11}$ I-CBT also facilitates self-monitoring of symptoms, is selfscheduled, and is not yoked to a setting, making it easily accessible to populations with treatment barriers. However, given that most trials evaluating the efficacy of I-CBT have used waitlist control comparators, and relative to face-to-face $\mathrm{CBT}$, Internet-based psychotherapy studies tend to exclude more severely ill patients, it's conceivable that patients who enter these trials are responding to non-specific treatment factors over time. Despite the hope of similar efficacy and greater accessibility and scalability, few studies have compared therapist-assisted I-CBT for PTSD to an active control condition, and none have focused on WTC responders or survivors-so its relative efficacy remains unclear. $^{12-14}$ To assess the efficacy of I-CBT, randomized controlled trials employing an active control condition are required. This is not only important for advancing the field of I-CBT interventions more broadly, but also for identifying empirically supported PTSD treatments that might facilitate treatment access for difficult-to-reach populations like the WTC cohort.

This report describes the design of an ongoing randomized controlled trial (RCT) of therapist-assisted ICBT, integrative testimonial therapy (ITT), for WTC responders and survivors with full or subthreshold WTCrelated PTSD, compared to a control intervention, internet-based modified present-centered therapy (IMPCT). ${ }^{11,12}$ This study is the first to evaluate the efficacy of ITT relative to an active control condition, and is the first to evaluate I-CBT in WTC responders and survivors. The primary aim is to evaluate the feasibility and efficacy of ITT in mitigating WTC-related PTSD symptoms in WTC responders and survivors. We hypothesize that ITT will be associated with clinically significant and moderate-to-large magnitude reductions in WTC-related PTSD symptoms (the primary outcome), assessed with the PTSD Checklist for DSM-5 (PCL-5), modified to inquire about WTC-related trauma symptoms, compared with I-MPCT. ${ }^{15}$ Secondary outcomes include comorbid depressive and anxiety symptoms, functioning, quality of life, and posttraumatic growth. We hypothesize that ITT will be associated with moderate-to-large magnitude improvements in functioning, quality of life, and posttraumatic growth, compared with I-MPCT.

Exploratory aims include evaluating baseline genetic and epigenetic predictors of treatment response and changes in epigenetic regulation of genes implicated in risk for PTSD from pre- to post-treatment. We expect that cumulative genetic and epigenetic risk factors for PTSD throughout the genome will predict response to treatment. We will i) construct polygenic risk scores (PRS) from the largest available PTSD genome-wide association study (GWAS) dataset, and test for association with treatment outcome, and ii) perform an epigenome-wide association study (EWAS), test for differential percent methylation, and identify specific differentially methylated positions (DMPs) between treatment responders and nonresponders.

\section{METHODS}

\section{Overview of study design and internet-based psychotherapy treatments}

Both Internet-based treatments contain three phases, comprising eleven narrative-based writing assignments ("narratives"), completed online via a secure web platform, over a 6-week treatment period. Patients are 
instructed to complete two 45-minute narratives weekly. Prior to each scheduled narrative, therapists at Mt. Sinai provide patients with written instructions. Therapist feedback is provided asynchronously (within 24-48 hours) for every 1-2 narratives and consists of standardized and personalized text addressing 1) individual patient content and 2) specific treatment phase goals within the framework of each intervention. Patient and therapist exchanges are conducted entirely online in writing, except in cases of safety concern or if a patient expresses confusion about the narrative instructions in which case the therapist calls the patient via telephone. This project, approved by the Icahn school of medicine at Mount Sinai IRB, is registered on ClinicalTrials.gov (NCT03154151). Patients are reimbursed up to $\$ 120$ for completion of study questionnaires and optional saliva collection.

\section{Integrative testimonial therapy (ITT)}

ITT is an internet-based, therapist-assisted, traumafocused CBT. It encompasses CBT elements including exploration of past and current challenges and coping resources, narrative exposure therapy, and cognitive reappraisal. ITT, initially developed for treating PTSD symptoms among elderly German world war II trauma survivors, was modified for the current study. ${ }^{11}$ ITT guides patients in writing a resource-oriented biographical reconstruction of their life, followed by exposure-based writing sessions focused on their WTCrelated traumatic exposures, and ending with cognitive reappraisal and reflective sessions. The intent is to integrate the patient's traumatic experiences into their broader life narrative, while supporting the patient's own adaptive coping strategies and challenging maladaptive thoughts and behavior.

\section{ITT treatment phases}

Resource-oriented biographical reconstruction (4 narratives) of the WTC responder/survivor's life, based on a collapsed version of the proposed psychosocial phases of Erikson and Erikson: ${ }^{16}$ (1) childhood (birth to age 12); (2) adolescence (ages 13-18); (3) early adulthood prior to the traumatic event (age 19-09/10/2001); and (4) life since the traumatic event (9/11/2001-to present). ${ }^{1}$ These phases were adapted for WTC survivors who were children or adolescents at the time of the 9/11/2001 WTC attacks. WTC survivors who were children or adolescents at the time of the $9 / 11$ attacks complete the first 1-2 biographical narratives followed by the 4 exposure sessions before completing the remaining 2 biographical narratives covering the development phases from 9/11/2001-present. In comparison, WTC responders, and many WTC survivors, who experienced WTC-related exposures in adulthood complete the first 3 biographical narratives followed by the 4 exposure sessions before completing the final biographical narrative focused on 9/11/2001-present. For each narrative, patients write about positive and negative events they encountered during the corresponding life phase, including hardships they faced, modes of coping they employed, and strengths they gained. The final biographical narrative, which takes place after the exposure narratives, asks patients to describe how their WTC-related trauma may influence their ability to act, respond, and behave in their present-day life. To encourage narrative reconstruction, guided instructions, historical memory cues, and typical, age-relevant developmental experiences are offered (i.e., childhood home, intimate relationships, changes in occupation, etc.).

Exposure (4 narratives) include detailed descriptions of patients' WTC-related traumatic experiences, including their most painful images and thoughts in the first person, as if the experiences were happening now. Patients are encouraged to include visual, auditory, and olfactory perceptions.

Integration: cognitive reappraisal (3 narratives) encourages a new perspective on the patient's WTC experience and promotes a sense of control over the trauma. Patients write successive drafts of a supportive letter to themselves at the time of their WTC experience from their current perspective as a trauma survivor. They are instructed to reflect on their feelings, to challenge maladaptive beliefs and behaviors, and to modify unrealistic assumptions. Patients are encouraged to reflect upon what they have learned throughout therapy, including coping strategies, and to continue to re-read their letters of support in the future.

Throughout treatment, therapist feedback acknowledges the patient's courage in disclosing and describing their traumatic experiences, reinforces completion of narratives, offers positive feedback, and encourages patients to voice any questions and doubts.

\section{Internet-based modified present-centered therapy (I- MPCT)}

Internet-based modified present-centered therapy (IMPCT), the control condition, is designed to assist WTC responders/survivors with PTSD in managing daily life stressors. I-MPCT parallels ITT in structure, mirroring treatment dose and frequency, and level of therapist interaction. I-MPCT features components of presentcentered therapy (PCT), a face-to-face supportive, present-day stressor-focused, problem-solving treatment. ${ }^{17}$ PCT was developed as a comparison condition in psychotherapy trials, but has since been cited as a research-supported intervention for PTSD. ${ }^{18,19}$ In an 8-week RCT assessing therapist-assisted, internet-based self-management CBT for PTSD, the therapist-assisted supportive counseling control condition featured components of PCT, including self-monitoring and writing about daily non-trauma related concerns. ${ }^{12}$ While the I-CBT intervention produced a significantly sharper decline in mean total PTSD symptom severity, associated with a small relative effect size difference $(\mathrm{d}=0.41)$, both 
conditions yielded a decline in mean total PTSD symptoms at post-treatment. In this study, I-MPCT retains PCT's rationale and goals, but does not include typical PCT homework assignments to match ITT procedures.

\section{I-MPCT treatment phases}

Getting to know you (3 narratives) prompts patients to write about themselves and their current circumstances (living situation, hobbies, important relationships, etc.). Therapist responses are designed to facilitate the patient's writing about meaningful aspects of their current life, establish rapport, and demonstrate empathy.

Identifying problems ( 4 narratives) guides the patient to identify and increase their awareness and understanding of current problem areas. The therapist encourages the patient to explore problems methodically and to identify thoughts and feelings that arise in response to challenges.

Problem solving in action (4 narratives) provides instructions for problem solving, brainstorming potential solutions, and writing about the pros/cons of each solution and potential challenges they might face. Therapist feedback seeks to foster the patient's sense of agency and control, while praising the patient's commitment to sharing difficult problems and working to generate potential solutions (e.g., identifying obstacles that impede attempting solutions).

While the painful impact of the patient's WTC experience is acknowledged by the therapist in the initial written introduction, I-MPCT emphasizes focusing on present-day circumstances. Consistent with PCT, there is no explicit writing or feedback about one's traumatic experience. Patients are not explicitly instructed to contextualize their current problems and stressors in reference to their WTC-related trauma or PTSD symptoms. If a patient does write about aspects of their WTC-related trauma or difficulties associated with their PTSD symptoms, the I-MPCT therapist offers empathy and validation. If a patient expresses confusion about or writes inaccurately about PTSD symptoms, the therapist provides corrective psychoeducation about PTSD. Such feedback in PCT intends to acknowledge what the patient is writing before redirecting their attention to the task of exploring current challenges. ${ }^{17}$

\section{Therapist training and supervision}

Four female therapists were selected for training in the online treatment procedures based on prior clinical training and experience working with WTC and other trauma survivors. A Doctoral-level social worker and a Master's-level clinical social worker received ITT training from co-investigators Drs. Knaevelsrud and Böttche, and receive ongoing supervision from Dr. Böttche. Two other Master's-level clinical social workers trained in I-MPCT by co-investigators Drs. Litz and
Presseau receive ongoing supervision from Dr. Presseau. Northeast tele-therapy requirements include licensure in the state in which the patient resides. All treating therapists are licensed in New York State, as well as either New Jersey or Pennsylvania, to randomize patients across treatment conditions in all three states. Quality control procedures include standardized treatment manuals, ongoing supervision, and fidelity monitoring to ensure adherence to treatment protocol and appropriate clinical care.

\section{Treatment fidelity}

For each therapist's first two cases, drafts of their written responses were first reviewed and discussed with their supervisor to shape treatment fidelity. Twenty percent of sessions (approximately 2 narrative exchanges) for each patient are selected from two of the three ITT and IMPCT phases using a random number generator to monitor fidelity at various treatment time points. Two fidelity raters trained in both online treatment manuals review the selected cases using guided fidelity checklists developed by each treatment condition's supervisor. Fidelity checks are divided into two sections: (1) adherence to treatment protocol via unique and fundamental elements specific to each session (i.e., describing feelings shared and summarizing narrative content); and (2) proscribed behavior (i.e., use of challenging or confrontational language, instruction to write about traumatic experience for I-MPCT cases). Each section includes 4-8 questions, and the rater gives a binary "Yes/No" rating as to whether each element is either present or absent. A case is considered adherent if there are zero occurrences of proscribed behavior and the percentage of adherent steps is at least $85 \%$. Approximately $15 \%$ of rated cases will be assessed for interrater reliability. Non-adherent cases are discussed with the project's data safety monitoring board to determine steps for corrective action (i.e., providing additional supervision to address therapist nonadherence).

\section{Patient recruitment and screening}

Sources of recruitment include: (1) contacting WTC responders and survivors either enrolled in the WTC-HP for health monitoring visits or registered with the WTC health registry; (2) community outreach; (3) clinician referral; and (4) print/web advertising to groups with large cohorts' of 9/11 responders and survivors (support groups, police department, etc). Individuals are directed to the project website (http://labs.icahn.mssm.edu/wtconlinestudy/ptsd/), which links to a pre-screening questionnaire and online consent form. Patients who sign the consent form electronically are emailed an online screening questionnaire including demographics, psychiatric and substance use history questions, a checklist of medical conditions, current medications, and a brief open-ended written description of the patient's experience during the WTC attacks and/or recovery 
work. A psychiatric telephone assessment is then conducted, including administration of the miniinternational neuropsychiatric interview (MINI) to complete the eligibility assessment. ${ }^{20}$ Eligible individuals receive a comprehensive review of study procedures via telephone, and ineligible individuals are provided alternative treatment options.

\section{Inclusion criteria}

Inclusion criteria included-(1) Male or female WTC responders or survivors with clinically significant WTCrelated PTSD symptoms on the PTSD Checklist (PCL-5), confirmed by the MINI, meeting criteria for (a) DSM-5 PTSD or (b) subthreshold PTSD, defined as either (i) 1 Criterion B (intrusion) symptom and either 3 Criterion C (avoidance) or Criterion D (negative mood and cognitions) or 2 Criterion $\mathrm{E}$ (arousal) symptoms, or (ii) 1 criterion $\mathrm{B}, 1$ either Criterion $\mathrm{C}$ or $\mathrm{D}$, and 1 criterion $\mathrm{E}$ symptom. For symptoms to be considered clinically significant, WTC responders/survivors meeting eligibility criteria must also report at least moderate functional impairment or distress resulting from trauma symptomatology in the past month. ${ }^{20-22}$

\section{Exclusion criteria}

(1) Elevated suicidal or homicidal risk; (2) psychotic symptoms, psychotic disorder, or bipolar disorder; (3) significant dissociative symptoms; (4) alcohol or drug use disorder within the previous 3 months; (5) current uncontrolled medical illness (e.g., cancer), neurological disorder affecting the central nervous system (e.g., multiple sclerosis), or history of head injury; (6) currently receiving psychotherapy; (7) currently taking antipsychotic medication, lithium or valproic acid. Other psychotropic medications (e.g., antidepressants, prazosin, hypnotic medications) are permitted at stable doses.

\section{Randomization}

After completing the pre-treatment questionnaire, patients are randomized to ITT or I-MPCT using an allocation sequence pre-generated by offsite co-investigator (RP). Treatment assignment remains concealed to enrollment personnel using sequentially numbered, opaque envelopes. Stratification ensures that equal numbers of traditional responders (e.g., police, firefighters, emergency medical technicians), non-traditional responders (e.g., construction workers, public transit employees, volunteers), and WTC survivors are allocated to each treatment arm. Patients are unaware of treatment assignment until receipt of their first online instructions.

\section{Assessment of treatment efficacy}

Primary and secondary outcomes are assessed at pretreatment baseline, post-treatment, and 3-month followup via online self-report questionnaires (see Table 1).

\section{Primary outcome: WTC-related PTSD symptoms}

The primary outcome measure is the total score on the PTSD Checklist-5 (PCL-5), a well-validated and widely used measure with excellent psychometric properties. ${ }^{15,23}$ Treatment response will be assessed using both (1) continuously distributed PCL-5 total scores, representing overall WTC-related PTSD symptom severity, and (2) a categorical response variable indicating clinically significant PTSD caseness (i.e., meeting vs. not meeting criteria for full or subthreshold WTC-related PTSD as defined in the inclusion criteria above). Treatment response reflects changes in PCL-5 scores from pretreatment at post-treatment and 3-months follow up.

\section{Secondary outcomes}

A comprehensive measure battery assesses secondary outcomes including efficacy of online treatments in improving functioning, quality of life, and posttraumatic growth, as well as reducing associated symptoms highly comorbid with PTSD (see Table 1).

Table 1: Assessment overview and timeline of primary and secondary outcome measures.

\begin{tabular}{|c|c|c|c|c|}
\hline Measures & $\begin{array}{l}\text { Pre- } \\
\text { Tx }\end{array}$ & Weekly & $\begin{array}{l}\text { Post } \\
-T x\end{array}$ & $\begin{array}{l}\text { 3MF } \\
\mathbf{U}\end{array}$ \\
\hline $\begin{array}{l}\text { PTSD checklist for } \\
\text { DSM-5 (PCL-5) }\end{array}$ & $\mathrm{X}$ & $X$ & $X$ & $\mathrm{X}$ \\
\hline $\begin{array}{l}\text { Beck depression } \\
\text { inventory-version II } \\
(\text { BDI-II })^{\mathbf{3 5}}\end{array}$ & $\mathrm{X}$ & $\mathrm{X}$ & $X$ & $\mathrm{X}$ \\
\hline $\begin{array}{l}\text { Generalized anxiety } \\
\text { disorder-7 (GAD-7) }\end{array}$ & $\mathrm{X}$ & $X$ & $\mathrm{X}$ & $\mathrm{X}$ \\
\hline $\begin{array}{l}\text { Medical outcomes } \\
\text { study (MOS) short } \\
\text { form } 8 \text { health survey } \\
(\text { MOS-SF-8) }\end{array}$ & $\mathrm{X}$ & & $X$ & $\mathrm{X}$ \\
\hline $\begin{array}{l}\text { Quality of life } \\
\text { enjoyment and } \\
\text { satisfaction scale- } \\
\text { short form (Q-LES- } \\
\text { SF) }\end{array}$ & $\mathrm{X}$ & & $X$ & $\mathrm{X}$ \\
\hline $\begin{array}{l}\text { Post-traumatic } \\
\text { growth inventory } \\
\text { short form (PTGI- } \\
\text { SF) }\end{array}$ & $\mathrm{X}$ & & $\mathrm{X}$ & $\mathrm{X}$ \\
\hline Biomarkers & $\mathrm{X}$ & & $\mathrm{X}$ & \\
\hline
\end{tabular}

\section{Assessment of participant safety and concomitant medications}

Periodic questionnaires are administered after the first 3 narratives and thereafter every 2 narratives to monitor patient safety, possible substance use, and potential changes in concomitant psychotropic medications. A staff member reviews patient responses and informs the 
therapist and study PI if any response suggests increased safety concern or a recent change in concomitant medication. Therapists remain alert to potential safety concerns raised by a patient's narrative content. In cases of safety concern the patient's therapist conducts a risk assessment via telephone. If a patient is judged to be a danger to self or others, they're exited from the study and provided with a referral for standard clinical care.

\section{Saliva sample collection and processing}

Patients who consent to specimen collection for biomarker assays are asked to provide saliva samples at pre-and post-treatment, collected via pre-paid mail. Saliva samples are collected at home, upon first awakening, using Genotek's Oragene-DNA (DNA 500) collection kits. Patients are provided with prepared, prestamped mailing materials to return collected samples.

Samples will be genotyped using the Infinium global screening array (GSA), the gold-standard genotyping array for cross-ancestry analyses. ${ }^{24}$ Methylation will be assessed using the Infinium MethylationEPIC BeadChip $\mathrm{Kit}^{24}$ which includes over 850,000 methylation sites genome-wide. We will perform stringent quality control for methylation and genotyping data using standard parameters and approaches. ${ }^{25,26}$

\section{Sample size considerations}

Prior I-CBT studies have observed large effect size (ES) improvements using waitlist control conditions, with Cohen's d's $>1.0 .^{8-10}$ Results from a Cochrane review of therapist-supported RCTs of I-CBT trials for anxiety disorders in adults (38 studies, 3,214 participants) revealed that, relative to waitlist, attention information, and online discussion group controls, I-CBT was associated with a large magnitude differential ES reduction in anxiety symptoms at post-treatment (i.e., $\mathrm{d}=1.06,95 \% \mathrm{CI}=0.82-1.29) .{ }^{27}$ However, the use of waitlist controls as a comparator poses limitations when evaluating ES estimates. A network meta-analysis examining differences among various control conditions in CBT psychotherapy trials for adult depression found treatment ES estimates differed substantively depending on the control condition employed; wait-list controls may generate larger ES estimates when compared to no treatment or psychological placebo. ${ }^{28}$ While differential ESs relative to waitlist controls must be considered, RCTs of I-CBT for PTSD that employed an active control treatment similarly observed large magnitude differential ES reductions in PTSD symptoms (d's $=0.95$ 1.25). ${ }^{12,29}$ Employing an active control condition, we anticipate more modest differential ES change in our primary outcome measure-PTSD Checklist-5 scores. ${ }^{21}$ Accordingly, based on differential ESs of prior Internet-based RCTs for PTSD and related anxiety disorders, we powered this trial to detect a minimum 0.80 magnitude differential ES change in PCL-5 scores from baseline between the ITT vs. I-MPCT treatment arms; this differential ES represents the lower end of the $95 \%$ confidence interval observed in the Cochrane review, and is 0.15-0.45 SDs lower than the differential ESs observed in two RCTs of I-CBT for PTSD. ${ }^{12,27,29}$ Thus, assuming a minimum differential 0.80 SD reduction in PCL-5 scores, power $=0.80, \alpha=0.05$, and correlation among repeated measures $=0.50$, recruiting 80 treatment completers $(n=40$ per treatment arm) will provide $>90 \%$ statistical power to detect differential ES magnitude changes as small as $\mathrm{d}=0.32$. We anticipate up to $20 \%$ dropout across conditions, and will accordingly randomize $n=100$ patients and use statistical techniques to account for early dropouts. ${ }^{11}$ Based on prior psychotherapy studies of trauma survivors with PTSD, candidate biomarkers evaluated in this study were moderate-to-large (i.e., d's=0.62-1.01) magnitude predictors of treatment response and measures of treatment effect. ${ }^{30-32}$

\section{Data analysis plan}

Linear mixed-effect models will be conducted to analyze treatment effects on all study outcome measures. Treatment (ITT vs. I-MPCT), time (post-intervention, three-month follow-up), treatment $\mathrm{x}$ time interaction, and past mental health treatment history will be entered as fixed factors; baseline scores and comorbidities (assessed at baseline and post-treatment) as fixed covariates, subject as a random effect, and scores on self-report measures (e.g., PCL-5) as dependent variables in separate analyses. Both intention-to-treat and treatment completer analyses will be conducted, computing treatment-related changes in WTC-related PTSD symptoms.

We will construct a PRS derived from the latest and largest GWAS statistics for PTSD. Briefly, PRS are constructed for each individual as a weighted sum of risk alleles within the genome. Weights used to construct these scores may be derived directly from GWAS summary statistics (i.e., may simply be the observed effect size in a study), or may be shrunken or scaled to optimize performance. We will follow the latest goldstandard approaches to maximize predictive accuracy of our scores. ${ }^{33}$ We will correct for gender, birth year, and ancestry (both self-reported and genotype-derived). We will apply a nested modelling approach to calculate the phenotypic variance explained by our polygenic score.

In addition to PRS-based analysis, will incorporate fixed effect (e.g., genotype) and interaction (e.g., treatment $\mathrm{x}$ genotype) terms into the linear mixed-effect and logistic regression models of symptom-based outcome measures. Only biomarkers that show bivariate associations with these measures at the $\mathrm{p}<0.20$ level will be entered.

We will perform an EWAS using genome-wide patterns of DNA methylation identified using the Infinium MethylationEPIC BeadChip Kit. ${ }^{24}$ We will identify DMPs in treatment-responders vs. non-responders and test whether these DMPs are enriched in specific 
regulatory regions or pathways. ${ }^{26}$ Will compare percent methylation in treatment-responders vs. non-responders.

\section{DISCUSSION}

I-CBT has demonstrated effectiveness in routine clinical practice and in clinical trials. ${ }^{11,34}$ Despite growing research bolstering the efficacy and feasibility of I-CBT in mitigating PTSD and related symptoms, few studies have compared therapist-assisted I-CBT for PTSD to an active control condition. We described the design of an ongoing RCT comparing therapist-assisted, I-CBTintegrative testimonial therapy (ITT)-to therapist-assisted, internet-based modified present-centered therapy (IMPCT) for WTC responders and survivors with full or subthreshold WTC-related PTSD. This RCT aims to evaluate the efficacy of a more accessible and scalable internet-based intervention for these unique populations.

It is possible that results may show no significant differences between ITT and I-MPCT, as both therapies may yield meaningfully lower WTC-related PTSD symptoms. I-MPCT aims to help patients better manage current problems and may lower WTC-related PTSD symptoms and functional impairment without directly addressing the WTC-related trauma. As a non-traumafocused therapy, I-MPCT might prove more tolerable than an exposure-based treatment like ITT and result in fewer treatment dropouts.

Every trial has methodological limitations. This RCT allows enrollment of individuals taking psychotropic medications. Although permitting concurrent psychotropic medications might confound treatment outcomes, this leniency enables more individuals to participate and increases the generalizability of findings. Second, the study's web platform requires access to a computer, the Internet, and having basic computer literacy skills. These criteria might exclude individuals from disadvantaged backgrounds who might not have access to such resources. However, in 2016, $72 \%$ and $82 \%$ of U.S. households reported having a desktop or laptop computer in the home and an Internet subscription, respectively. While percentages specific to the WTC cohort are unknown, the presence of computers and Internet access in U. S. households is encouraging for the dissemination of Internet-based treatments. While PTSD diagnosis or the presence of subthreshold PTSD were determined via direct interview with the MINI, the primary and secondary outcome assessments are based on self-report questionnaires. The addition of clinicianadministered ratings to confirm self-report responses is warranted in future trials.

\section{CONCLUSION}

This study addresses an unmet need for mental health treatment among an in-need population of WTC responders and survivors who continue to suffer clinically significant WTC-related PTSD symptoms and functional impairment. This RCT bridges an important research gap in the rising field of I-CBT interventions. Findings from this trial will contribute to the literature on the development of new evidence-based treatments for PTSD in WTC- and other trauma-affected populations.

\section{ACKNOWLEDGEMENTS}

Authors would like to thank the WTC health program faculty, administrative staff, and WTC responders and survivors for their invaluable cooperation and support. We are especially grateful to the WTC data center, WTC health registry, and other groups and volunteer organizations working with WTC responders and survivors that have significantly contributed to recruitment efforts for this study and have made this project possible.

Funding: Funding sources CDC/National institute for occupational safety and health (NIOSH) grant UOI OHO 10729.

Conflict of interest: Dr. Feder is named co-inventor on an issued patent in the United States, and several issued patents outside the U.S., filed by the Icahn School of Medicine at Mount Sinai (ISMMS) for the use of ketamine as a therapy for PTSD. This intellectual property has not been licensed. The other authors have no disclosures to report.

Ethical approval: The study was approved by the Institutional Ethics Committee of Icahn school of medicine at Mount Sinai IRB and was registered on ClinicalTrials.gov on May 16, 2017 (NCT03154151).

\section{REFERENCES}

1. Feder A, Mota N, Salim R. Risk, coping and PTSD symptom trajectories in World trade center responders. J Psychiatr Res. 2016;82:68-79.

2. Jordan HT, Osahan S, Li J. Persistent mental and physical health impact of exposure to the September 11, 2001 World Trade Center terrorist attacks. Environ Health. 2019;18(1):12.

3. Chen C, Salim R, Rodriguez J. The Burden of Subthreshold Posttraumatic Stress Disorder in World Trade Center Responders in the Second Decade After 9/11. J Clin Psychiatry. 2020;81(1).

4. Farfel M, DiGrande L, Brackbill R. An overview of 9/11 experiences and respiratory and mental health conditions among World Trade Center Health Registry enrollees. J Urban Health. 2008;85(6):880909.

5. Jacobson MH, Norman C, Sadler P, Petrsoric LJ, Brackbill RM. Characterizing Mental Health Treatment Utilization among Individuals Exposed to the 2001 World Trade Center Terrorist Attacks 14-15 Years Post-Disaster. Int $\mathrm{j}$ environmental res pub health. 2019;16(4):626.

6. Markowitz JC, Milrod B, Heckman TG. Psychotherapy at a Distance. Ame J Psychiatry. 2020 . 
7. Wang PS, Berglund P, Olfson M, Pincus HA, Wells $\mathrm{KB}$, Kessler RC. Failure and delay in initial treatment contact after first onset of mental disorders in the National Comorbidity Survey Replication. Arch Gen Psychiatry. 2005;62(6):603-13.

8. Knaevelsrud C, Maercker A. Internet-based treatment for PTSD reduces distress and facilitates the development of a strong therapeutic alliance: a randomized controlled clinical trial. BMC Psychiatry. 2007;7:13.

9. Knaevelsrud C, Liedl A, Maercker A. Posttraumatic growth, optimism and openness as outcomes of a cognitive-behavioural intervention for posttraumatic stress reactions. J Health Psychol. 2010;15(7):10308.

10. Wagner B, Schulz W, Knaevelsrud C. Efficacy of an Internet-based intervention for posttraumatic stress disorder in Iraq: a pilot study. Psychiatry Res. 2012;195(1-2):85-8.

11. Knaevelsrud C, Bottche M, Pietrzak RH, Freyberger HJ, Renneberg B, Kuwert P. Integrative testimonial therapy: an Internet-based, therapist-assisted therapy for German elderly survivors of the World War II with posttraumatic stress symptoms. J Nerv Ment Dis. 2014;202(9):651-8.

12. Litz BT, Engel CC, Bryant RA, Papa A. A randomized, controlled proof-of-concept trial of an Internet-based, therapist-assisted self-management treatment for posttraumatic stress disorder. Am J Psychiatry. 2007;164(11):1676-83.

13. Spence J, Titov N, Johnston L, Jones MP, Dear BF, Solley K. Internet-based trauma-focused cognitive behavioural therapy for PTSD with and without exposure components: a randomised controlled trial. J Affect Disord. 2014;162:73-80.

14. Steinmetz SE, Benight CC, Bishop SL, James LE. My Disaster Recovery: a pilot randomized controlled trial of an Internet intervention. Anxiety Stress Coping. 2012;25(5):593-600.

15. Blevins CA, Weathers FW, Davis MT, Witte TK, Domino JL. The posttraumatic stress disorder checklist for DSM-5 (PCL-5): Development and initial psychometric evaluation. $\mathrm{J}$ traumatic stress. 2015;28(6):489-98.

16. Erikson EH, Erikson JM. The life cycle completed (extended version). WW Norton and Company. 1998.

17. Bernardy N, Davis N, Howard J, Key F, Lambert J, Shea MT. Present centered therapy manual (Unpublished therapy manual). 2008.

18. Imel ZE, Laska K, Jakupcak M, Simpson TL. Metaanalysis of dropout in treatments for posttraumatic stress disorder. J Consult Clin Psychol. 2013;81(3):394-404.

19. Resick PA, Wachen JS, Mintz J. A randomized clinical trial of group cognitive processing therapy compared with group present-centered therapy for PTSD among active duty military personnel. J Consult Clin Psychol. 2015;83(6):1058-68.
20. Sheehan DV, Lecrubier Y, Sheehan KH. The MiniInternational Neuropsychiatric Interview (M.I.N.I.): the development and validation of a structured diagnostic psychiatric interview for DSM-IV and ICD-10. J Clin Psychiatry. 1998;59(20:22-33):34-57.

21. Weathers F, Litz B, Herman D, Huska J, Keane T. The PTSD Checklist (PCL): reliability, validity, and diagnostic utility. Ann Convention Int Society Traumatic Stress Studies. 1993;TX.

22. McLaughlin KA, Koenen KC, Friedman MJ. Subthreshold posttraumatic stress disorder in the world health organization world mental health surveys. 2015;77(4):375-84.

23. Bovin MJ, Marx BP, Weathers FW. Psychometric properties of the PTSD Checklist for Diagnostic and Statistical Manual of Mental Disorders-Fifth Edition (PCL-5) in veterans. Psychol Assess. 2016;28(11):1379-91.

24. Available at: https://www.illumina.com/products/bytype/microarray-kits/infinium-methylation-epic.html. Accessed on 2 March, 2021.

25. Coleman JR, Euesden J, Patel H, Folarin AA, Newhouse S, Breen G. Quality control, imputation and analysis of genome-wide genotyping data from the Illumina HumanCoreExome microarray. Briefings functional genomics. 2016;15(4):298-304.

26. Hannon E, Dempster E, Viana J. An integrated genetic-epigenetic analysis of schizophrenia: evidence for co-localization of genetic associations and differential DNA methylation. Genome biol. 2016;17(1):1-16.

27. Olthuis JV, Watt MC, Bailey K, Hayden JA, Stewart SH. Therapist-supported Internet cognitive behavioural therapy for anxiety disorders in adults. Cochrane Database Syst Rev. 2016;3:CD011565.

28. Furukawa TA, Noma H, Caldwell DM. Waiting list may be a nocebo condition in psychotherapy trials: a contribution from network meta-analysis. Acta Psychiatr Scand. 2014;130(3):181-92.

29. Ivarsson D, Blom M, Hesser H, Carlbring $P$, Enderby P, Nordberg R, Andersson G. Guided internet-delivered cognitive behavior therapy for post-traumatic stress disorder: a randomized controlled trial. Internet Interventions. 2014;1(1):3340.

30. Bryant RA, Felmingham KL, Falconer EM. Preliminary evidence of the short allele of the serotonin transporter gene predicting poor response to cognitive behavior therapy in posttraumatic stress disorder. Biol Psychiatry. 2010;67(12):1217-9.

31. Felmingham KL, Dobson-Stone C, Schofield PR, Quirk GJ, Bryant RA. The brain-derived neurotrophic factor Val66Met polymorphism predicts response to exposure therapy in posttraumatic stress disorder. Biol Psychiatry. 2013;73(11):1059-63.

32. Yehuda R, Daskalakis NP, Desarnaud F. Epigenetic Biomarkers as Predictors and Correlates of Symptom Improvement Following Psychotherapy in Combat Veterans with PTSD. Front Psychiatry. 2013;4:118. 
33. Choi SW, Mak TS-H, O'Reilly PF. Tutorial: a guide to performing polygenic risk score analyses. Nature Protocols. 2020:1-14.

34. Ruwaard J, Lange A, Schrieken B, Emmelkamp P. Efficacy and effectiveness of online cognitive behavioral treatment: a decade of interapy research. Stud Health Technol Inform. 2011;167:9-14.

35. Beck AT WC, Mendelson M, Mock J, Erbaugh J. An inventory for measuring depression. Arch Gen Psychiatry. 1961;4(4):561-71.

36. Spitzer RL, Kroenke K, Williams JB, Lowe B. A brief measure for assessing generalized anxiety disorder: the GAD-7. Arch Intern Med. 2006;166(10):1092-7.

37. Turner-Bowker DM, Bayliss MS, Ware JE Jr, Kosinski M. Usefulness of the SF-8 Health Survey for comparing the impact of migraine and other conditions. Qual Life Res. 2003;12(8):1003-12.
38. Endicott J, Nee J, Harrison W, Blumenthal R. Quality of Life Enjoyment and Satisfaction Questionnaire: a new measure. Psychopharmacol Bull. 1993;29(2):321-6.

39. Cann A, Calhoun LG, Tedeschi RG. A short form of the Posttraumatic Growth Inventory. Anxiety Stress Coping. 2010;23(2):127-37.

Cite this article as: Brinkman HR, Kowalchyk ML, Cahn L, Aaronson CJ, Böttche M, Presseau C, Markowitz JC, Litz BT, Huckins L, Yehuda R, Knaevelsrud C, Pietrzak RH, Feder A. Internet-based cognitive behavioral therapy versus internet-based modified present-centered therapy for world trade center responders and survivors with posttraumatic stress disorder: rationale and design of a randomized controlled trial. Int J Clin Trials 2021;8(3):202-10. 